\title{
Should the Interest Rate Really Be the Unique Motive to Save in the Ramsey Model?
}

\author{
Atef Khelifi ${ }^{1}$ \\ ${ }^{1}$ CLERSE, Université de Lille 1, Villeneuve d'Ascq, France \\ Correspondence: Atef Khelifi, CLERSE, Université de Lille 1, 59650 Vileneuve d'Ascq, France. Tel: \\ 33-604-654-307. E-mail: atef.khelifi@ed.univ-lille1.fr
}

Received: June 6, 2016

Accepted: October 12, 2016

Online Published: November 25, 2016

doi:10.5539/ijef.v8n12p224

URL: http://dx.doi.org/10.5539/ijef.v8n12p224

\begin{abstract}
By assuming that the individual derives utility from consumption only, the resulting optimal decision to save in the Ramsey model depends on the rate of return, given a certain time preference. If therefore the production function is such that this rate of return remains relatively low, the individual reacts unconsciously by refusing to save despite the capital depreciates and the household grows. We argue that it is conceptually necessary in that framework to assume a direct preference for saving (or for thriftiness) in the utility function, not only to make the individual behave as a real human being who cares about the survival of the household, but also to account reasonably for any other motives to save or accumulate than the rate of return. We show it generalizes the model in a way to recover static properties of the exogenous Solow version and to extend results of capitalist spirit models following Zou (1994).
\end{abstract}

Keywords: bequest, status, thriftiness, capitalist spirit, ramsey model

\section{Introduction}

It goes to our mind almost systematically, that the flow of saving should never be included in the Utility function along with consumption, to solve the inter-temporal maximization problem of the consumer. Two reasons can be stated precisely and may sound a bit obvious only at first glance. The first one which is to consider savings as having no intrinsic value for the individual, can arguably be contested in some cases. The second, a technical redundancy applying to the basic model where the goal of the individual is to reallocate exogenous flows of income, can be shown to not apply to models of accumulation. The goal of this paper is to criticize the systematic neutrality of a Utility effect of savings in the literature, and to report the relevant implications in the Ramsey growth model where specifically, such neutrality may actually be inappropriate.

To contest the first reason for excluding savings from Utility in the Ramsey context, it might not be necessary to recall the literature motivating that individuals derive direct Utility from wealth for the status; see Zou (1994), Bakshi and Chen (1996), Carroll (2000) or Kumhof, Rancière and Winant (2015). Indeed, by introducing the saving flow in the Utility function, not only is the continual seek for a higher capital and status captured, but also a direct preference for thriftiness which should be involved if the individual worries about the necessity to renovate the depreciated capital, or to accumulate sufficient wealth for the growing household. (Note 1).

In life-cycle (LC) or overlapping generations (OLG) models, the neutrality of this direct preference for thriftiness or wealth accumulation is systematically constrained each period, such that the individual saves only if the interest rate is attractive. Because of this particularity, alternative models accounting for the bequest motive have been proposed in the literature. One type includes for example Barro (1974) or Laitner (1992), where successive generations working for only one period value the level of Utility of their children. Another consists in "joy of giving models" like for example Andreoni (1990), or more recently Dynan, Skinner, and Zeldes (2002), where individuals gain satisfaction from bequeathing a part their lifetime income to the children. Although the second type has been extensively examined in the literature, the altruistic Ramsey model has never been explored under the assumption of a direct preference for bequeathing savings that are reinvested. It could be supposed indeed, that each generation working at period $t$ leaves a bequest that serves to renovate or increase the capital left to the children at $t+1$, before retiring or eventually dying. From this conceptual viewpoint, a direct preference for saving finds a very strong motivation in that framework, aside from a plausible preference for thriftiness or wealth accumulation. 
Technically, it may have seemed redundant at first glance to introduce explicitly this preference in the model, because the dynamics is already such that savings and accumulation occur up to a steady-state. However, this result is precisely due to the properties of the production function and not to a particular intention of the individual to accumulate or renovate capital. It is well known for example that under the case of a small open economy facing a relatively low world interest rate, the dynamics conterfactually predicts that this economy never saves and for instance, that it asymptotically mortgages all of its capital and labor income; see Barro and Sala-i-Martin (1995, chapter 3) or Hof and Wirl (2008). Similar unplausible results occur in the case of a closed economy with for example an $A k$ production function. Starting indeed from a steady-state position, if a permanent productivity shock occurs such that $A$ decreases to $A^{\prime}$, individuals chose to remain on a relatively high level of consumption and to neglect the capital depreciation until it totally disappears. We show (in Appendix) that including a direct preference for saving or for thriftiness in the utility function is a way to make the representative agent conscious as a real human being and to reach a new viable solution.

Another technical detail that defends the necessity to introduce a direct preference for saving in the Ramsey model is that, contrary to basic inter-temporal models where the goal of the individual is to reallocate exogenous flows of income, this one endogenizes the behavior of a 'saver' (the Solow agent); i.e. it studies the long-run accumulation process of capital that results from optimal demands for consumption and savings at each period. As a direct consequence, the microeconomic theorem of integrability applies, which means the corresponding Utility function is of the type $\mathrm{U}\left(\mathrm{C}_{\mathrm{t}}, \mathrm{S}_{\mathrm{t}}\right)$. (Note 2).

Assuming a direct preference for saving or accumulating generates a more general dynamics than the standard one and even those of models accounting for the capitalist spirit as Zou (1994). Furthermore, it allows to recover the basic static properties of the exogenous Solow version.

Similarly to growth models involving absolute wealth in Utility as Zou (1994), or relative wealth as Corneo and Jeanne (2001), the presented model allows to invest more than in the standard version by modulating the preference for wealth accumulation. This property is known as a plausible way to contrast with the contested lower boundary condition and convergence theorem of the traditional theory. It implies for instance that necessary and sufficient conditions required to meet the golden rule of accumulation can be specified. Another similarity that might also be important to report, is that the effect of the natural growth rate of workers on the steady-state level of capital per capita appears confirmed. Those common results could eventually be interpreted as two steps already made towards a reconciliation with Solow's static properties of the steady-state.

In contrast with this previous literature however, the proposed preference function generates a slower transition, and offers the possibility to reach also lower steady-state levels of capital per capita than in the standard model by investing less. Aside from allowing to recover a total coherence with the basic exogenous version, this second property might complement explanations of cross-country differences, and for instance, reconcile more empirical growth facts of developing countries with optimal growth theory.

The remainder is organized as follows. Section 2 shows that applying this type of preferences in the Ramsey model appears to generate more consistent results than in the standard case. Section 3 concentrates on a discussion of the transition through a comparative analysis, and Section 4 concludes.

\section{A Further Formulation of the Ramsey Growth Model}

The first section presents the model which involves an interesting application of the Pontryagin's Maximum Principle. The second discusses the steady-state and Golden Rule.

\subsection{The Optimal Control Program}

At time $t$, a representative generation composed of $\mathrm{L}$ workers cares about consuming and reinvesting a part of income produced. The instantaneous preference function is defined by $U_{t}\left(c_{t}, s_{t}\right)$, where $c_{t}$ denotes per capita consumption at $\mathrm{t}, s_{t}$ denotes per capita savings (or bequests), and $\theta \in(0,1)$ is a proportion which measures the degree of preference for consumption over savings. The budget constraint of this representative agent is given by $c_{t}+s_{t}=y_{t}$, where $y_{t}$ denotes income per worker. The production function is supposed of the Cobb-Douglas form with constant returns to scale, such that $y_{t}=f\left(k_{t}\right)=k_{t}^{\alpha}$, where $k_{t}$ denotes capital per worker, and $\alpha \in(0,1)$. The dynastic Utility function (after substitution of $s_{t}$ ) is denoted by $V\left\{c_{t}, k_{t}\right\}$. It is maximized over an infinite horizon subject to a dynamic constraint of capital accumulation (by the social planner):

$$
\left\{\begin{array}{l}
\operatorname{Max}_{c_{t}} V\left\{c_{t}, k_{t}\right\}=\int_{t_{0}}^{\infty} U_{t}\left(c_{t}, k_{t}\right) e^{(n-\beta) t} d t \\
\text { s.t. } \quad \dot{k}_{t}=f\left(k_{t}\right)-c_{t}-(n+\delta) k_{t}
\end{array}\right.
$$


where $n$ is the natural growth rate of workers, $\beta$ is the usual degree of impatience and $\delta$ is the rate of depreciation of capital. We impose the usual restriction $\beta>n$ to ensure a feasible interior solution to the problem.

For the sake of concreteness, we assume a Cobb-Douglas Utility function $U_{t}\left(c_{t}, s_{t}\right)=c_{t}^{\theta} s_{t}^{1-\theta}$ and its $\log$-transformation $U_{t}\left(c_{t}, s_{t}\right)=\theta \ln \left(c_{t}\right)+(1-\theta) \ln \left(s_{t}\right)$.

Considering the first specification for example, the Hamiltonian is given by:

$$
H_{t}\left(c_{t}, s_{t}\right)=c_{t}^{\theta}\left[f\left(k_{t}\right)-c_{t}\right]^{1-\theta} e^{(n-\beta) t}+\lambda_{t} e^{(n-\beta) t}\left[f\left(k_{t}\right)-c_{t}-(n+\delta) k_{t}\right]
$$

After standard computations (exposed in appendix), the resulting differential equation of consumption is:

$$
\dot{c}_{t}=\frac{1-a \theta}{1-\theta}\left[(2-a \theta-\theta) f_{k}-(1-a)(\delta+\beta)\right] c_{t}-a \theta(n+\delta) f_{k} k_{t}
$$

In clear, there can be multiple dynamical systems depending on the value of $a \in(0,1)$, which satisfy the first necessary conditions. This means that among all admissible values of $a$, or $\lambda_{t}$ as explained by Schättler and Ledzewicz (2012), it remains to determine which one(s) maximize(s) $V_{t}\left\{c_{t}^{*}, k_{t}^{*}\right\}$. In that sense, we may now define $a$ as being a choice variable associated to a set of controlled trajectories, and $a^{*}$ as being the rational choice associated to the optimal one which maximizes total welfare; i.e., the value of $a$ is endogenously set by the maximizing behavior.

Contrary to the differential equation of consumption (4), the one of the log-transformed Utility can admit the value of $\theta=1$, in which case it simplifies to the standard Ramsey equation. Its expression is given by:

$$
\frac{c_{t}}{c_{t}}=\frac{1-a \theta}{1+a \theta(a-2)}\left[2 a(1-\theta) f_{k}+(1-a)\left(f_{k}-\beta-\delta\right)\right]-\frac{a(1-\theta)}{1+a \theta(a-2)}(n+\delta) \alpha
$$

The graphical resolution of the dynamical systems shows that for any $a \in(0,1)$, there exists a unique saddle path in each case leading to a steady state equilibrium denoted by $\left\{c_{T}^{*}(a), k_{T}^{*}(a)\right\}$. The phase diagram corresponding to the multiplicative Cobb Douglas case (Figure 1), shows that the parameter a affects only the convexity of the ' $\dot{c}_{t}=0$ locus'. (Note 3 ) The level of consumption increases to the left of this locus, and decreases to the right. For the case of the log-Utility (Figure 2), the phase diagram is identical to the standard one except that the vertical ' $\dot{c}_{t}=0$ locus' depends now on the parameter $a$.

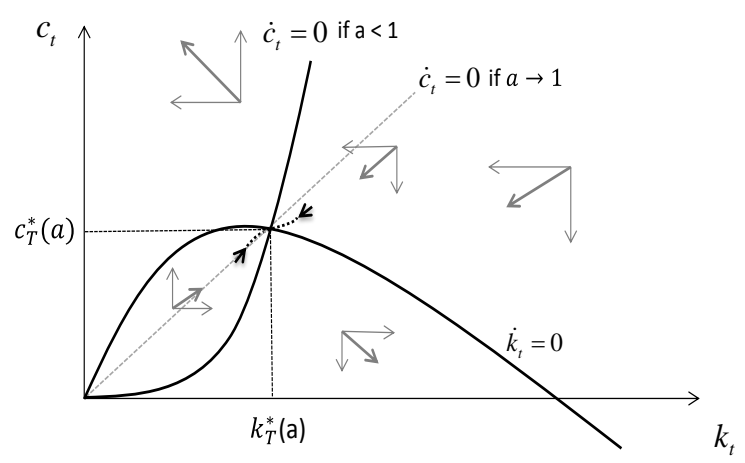

Figure 1. Phase diagram for the multiplicative Cobb-Douglas case

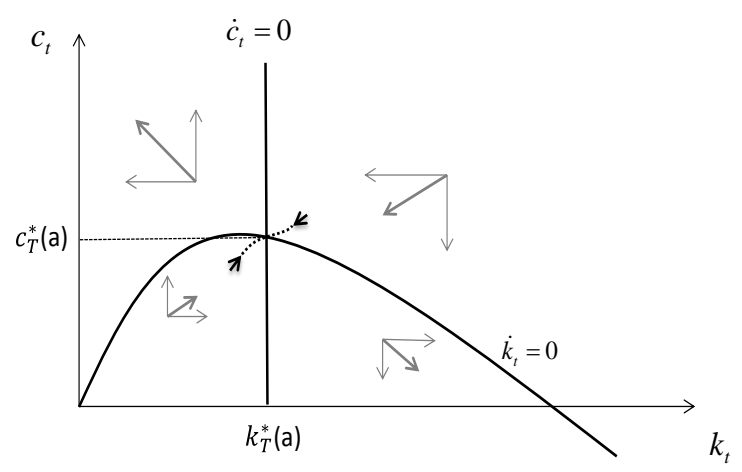

Figure 2. Phase diagram for the additive Cobb-Douglas case 
Let $\left\{c_{t}^{*}(a), k_{t}^{*}(a)\right\}$ denote any equilibrium (or saddle path) solution that leads to the steady-state at $t=T$. It can easily be noticed that the transversality condition $\lim _{t \rightarrow \infty} \lambda_{t}(a) k_{t}^{*}(a) e^{(n-\beta) t}=0$, is fulfilled at equilibrium if $\beta>n$ because:

$$
\left\{\begin{array}{l}
\lim _{t \rightarrow \infty} \lambda_{t}(a)=U_{c_{T}^{*}(a)}, \text { from the first necessary condition, } \\
\lim _{t \rightarrow \infty} k_{t}^{*}(a) e^{(n-\beta) t}=k_{T}^{*}(a) \cdot \lim _{t \rightarrow \infty} e^{(n-\beta) t}=0, \text { if } \beta>n
\end{array}\right.
$$

Let the control set be defined by:

$$
Z=\left\{c_{t}^{*}(a) \in \mathbb{R}^{++} / c_{t}^{*}(a)<y_{t}^{*}(a) \forall a \in(0,1)\right\},
$$

such that total welfare $V_{t}\left\{c_{t}^{*}(a), k_{t}^{*}(a)\right\}$ is restricted to the set of real numbers. We can now proceed to a formal definition of a feasible optimal solution to the problem.

PROPOSITION 1: An admissible controlled trajectory $\left\{c_{t}^{*}\left(a^{*}\right), k_{t}^{*}\left(a^{*}\right)\right\}$ satisfying the necessary Pontryagin's conditions $\forall a^{*} \in(0,1), c_{t}^{*}\left(a^{*}\right) \in Z$ and $k_{t}^{*}\left(a^{*}\right) \in \mathbb{R}^{++}$, is an optimal controlled trajectory if and only if $V_{t}\left\{c_{t}^{*}(a), k_{t}^{*}(a)\right\} \leq V_{t}\left\{c_{t}^{*}\left(a^{*}\right), k_{t}^{*}\left(a^{*}\right)\right\} \forall a \in(0,1) / a \neq a^{*}, c_{t}^{*}(a) \in Z, k_{t}^{*}(a) \in \mathbb{R}^{++}$.

An important result to keep in mind, is that the rational choice of the optimal trajectory is determinant for the terminal steady-state $\left\{c_{T}^{*}\left(a^{*}\right), k_{T}^{*}\left(a^{*}\right)\right\}$ reached in the long run.

\subsection{Steady-States and Golden Rule}

Contrary to the multiplicative Cobb Douglas Utility function, the $\log$ form allows to derive a steady-state solution analytically, which is:

$$
k_{T}^{*}(a)=\left[\frac{(1-a \theta)(1-2 a \theta+a) \alpha}{a(1-\theta)(n+\delta) \alpha+(1-a \theta)(1-a)(\beta+\delta)}\right]^{\frac{1}{(1-\alpha)}}
$$

For any non-corner point $(\theta, a) \in(0,1) \times(0,1)$, the steady-state level of capital (or income) per worker is a decreasing function of the natural growth rate. Hence, as soon as individuals are assumed to gain some Utility from accumulating or saving, the negative impact of population growth known from the basic exogenous Solow model is recovered.

Another important static property that plays a crucial role for the dynamics is the one of the golden rule from Phelps (1961). The Ramsey growth model, as it is used in most macroeconomic studies, is characterized by a steady-state level of capital per worker that remains always lower than the consumption maximizing level (and hence, than the over-accumulation one as well). As explained previously, this constitutes one of the reasons why alternative capitalist spirit models have been proposed in the literature, following for example Zou (1994) and Corneo and Jeanne (2001). Before presenting necessary and sufficient conditions for a golden rule steady-state, it may be preferable to present first a more general property that concerns any 'terminal' or constrained steady-state.

PROPOSITION 2: Among all feasible controlled trajectories $\left\{c_{t}^{*}(\theta, a), k_{t}^{*}(\theta, a)\right\} \in Z \times \mathbb{R}^{++}$, converging to a particular steady-state solution $\left\{\overline{C_{T}^{*}}, \overline{k_{T}^{*}}\right\}$, there exists a preference-choice couple $\left(\theta^{*}, a^{*}\right) \in(0,1) \times(0,1)$ compatible with an optimizing behavior; ie. which satisfies: $V_{t}\left\{c_{t}^{*}(\theta, a), k_{t}^{*}(\theta, a)\right\} \leq V_{t}\left\{c_{t}^{*}\left(\theta^{*}, a^{*}\right), k_{t}^{*}\left(\theta^{*}, a^{*}\right)\right\}$, $\forall(\theta, a) \in(0,1) \times(0,1) /(\theta, a) \neq\left(\theta^{*}, a^{*}\right)$.

This property resulting directly from the resolution might be viewed as a reciprocal of the first one. Indeed, a steady-state dynamics is an optimal controlled trajectory as soon as there is no other ways to reach the same dynamics with a higher total welfare. Suppose for example that the constrained terminal state corresponds to the solution of the Solow model:

$$
\overline{k_{T}^{*}}=\left(\frac{\overline{s_{T}^{*}}}{n+\delta}\right)^{\frac{1}{1-\alpha}} \text { where } f_{\overline{k_{T}^{*}}}=\frac{(n+\delta) \alpha}{\overline{s_{T}^{*}}} \text { and } \overline{s_{T}^{*}} \in(0,1)
$$

The determination of $\theta$ a posteriori for a given $\overline{s_{T}^{*}}$, requires to solve a quadratic equation in $\theta$, which implies two admissible sets $\left(\theta_{i}, a_{i}\right), \forall i=1,2$. In some cases, restrictions imposed allow to eliminate one of the sets entirely, so that a unique underlying combination of parameters $\left(\theta_{i}^{*}, a_{i}^{*}\right)$ that maximizes total welfare can be identified; it is for example the case for relatively high or low steady-states. Under cases where both sets offer potential candidates however, the terminal state assumed can possibly be generated by two different optimal trajectories (or two different rational behaviors). In such a multiple equilibrium context, the goodness of fit to 
real data becomes the last way to identify the right solution.

The golden rule steady-state which maximizes consumption is reached if $\overline{s_{T}^{*}}=\alpha$. For the multiplicative Cobb-Douglas form, the following condition must hold:

$$
\left[1+\frac{1-\theta}{(1-a \theta) X} a \theta(n+\delta)\right]^{-1}=\alpha
$$

where $X=(2-a \theta-\theta)(n+\delta)-(1-a)(\delta+\beta)$, and for the log-Utility case,

$$
\frac{1-a \theta}{a(1-\theta)}\left[1+a(1-2 \theta)-(1-a) \frac{\beta+\delta}{n+\delta}\right]=\alpha
$$

\section{Numerical Analysis of the Dynamics}

\subsection{General Properties of the Dynamics}

The question that comes first is to know how total welfare changes with respect to the parameter a, which has been defined previously as reflecting the choice of an admissible trajectory made by the individual. The next interesting step is to understand how the variables behave along the optimal path (Note 4).

Numerical simulations show that for a preference parameter $\theta$ that tends to one, the optimal value of $a$ decreases and the maximum total welfare tends to stabilize for $a<a^{*}$. In Figure 3 for example, when $\theta=0.95$, the maximum total welfare attains $V^{*}=241.62$ at $a^{*}=0.71$, and remains almost constant for $a \leq 0.71$ (precisely, it decreases slowly until $V=241.36$ for $a=0.01$ ). As $\theta$ decreases, the value of $a^{*}$ increases but at a much lower rate; for instance $a^{*}=0.76$ when $\theta=0.85$ and $a^{*}=0.78$ when $\theta=0.2$. This Figure shows also that the choice of the right equilibrium co-state (or value of a) matters much more for lower values of $\theta$. For example, the size of the welfare gain from an admissible path to the optimal one can exceed $26 \%$ for the case where $\theta=0.2$.

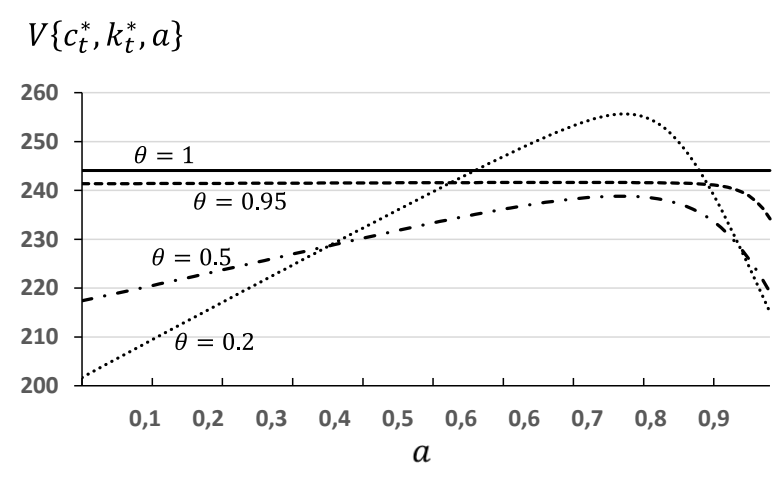

Figure 3. Total welfare by admissible path

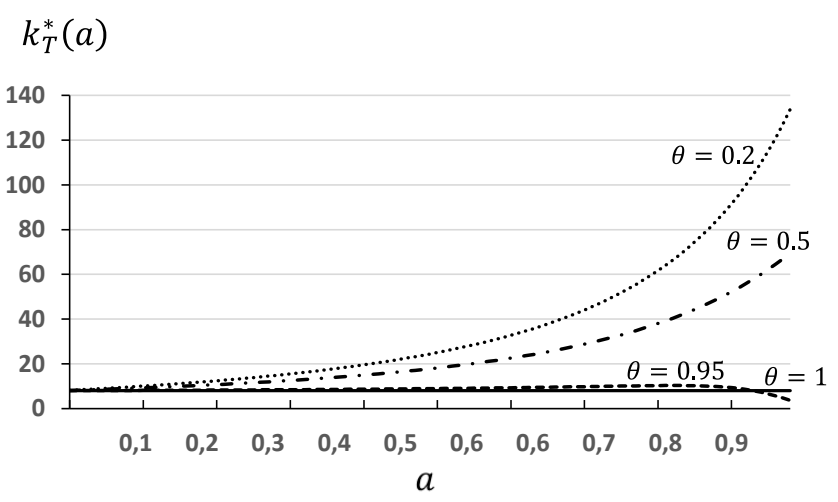

Figure 4. Steady-state per capita capital by admissible path

Another result that Figure 3 illustrates, is that individuals with different degrees of preference for saving face 
different levels of total welfare. When $\theta$ remains relatively high, total welfare is lower than in the standard case where individuals value consumption only. As $\theta$ decreases below a certain cutoff value, total welfare tends to increase back until reaching higher values than in the standard model. Besides the fact that the steady-state saving rate for such values of $\theta$ seems unreasonable, as indicated by Figure 6, this parameter describing fixed preferences is conceptually not to be 'selected' so that total welfare or even consumption is maximized. (Note 5).

In Figure 4, it is interesting to notice that accounting for supplementary motives for saving, does not always mean a higher steady-state capital per capita than in the standard model. For values of $\theta$ that are close to one (0.95 in our example), there are some admissible trajectories (for high values of a) which lead to lower steady-state capital per capita than in the standard case. This interesting remark will be developed later.

Concerning transitions towards higher-steady-states, Figure 5 shows that (unconstrained) optimal trajectories exhibit a faster growth when preferences for saving for social reasons become more intense. Interestingly, although the speed of growth increases with such intense preferences, thrifty individuals appear less sensitive to variations of the interest rate compared to those who care about consumption only. For instance, Figure 6 shows that the magnitude of the variation of the saving rate along the optimal path increases with $\theta$.

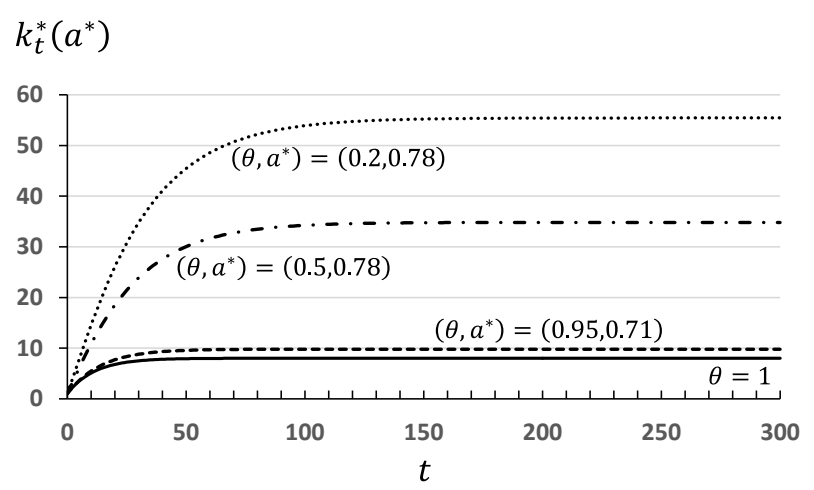

Figure 5. Equilibrium path of capital per capita

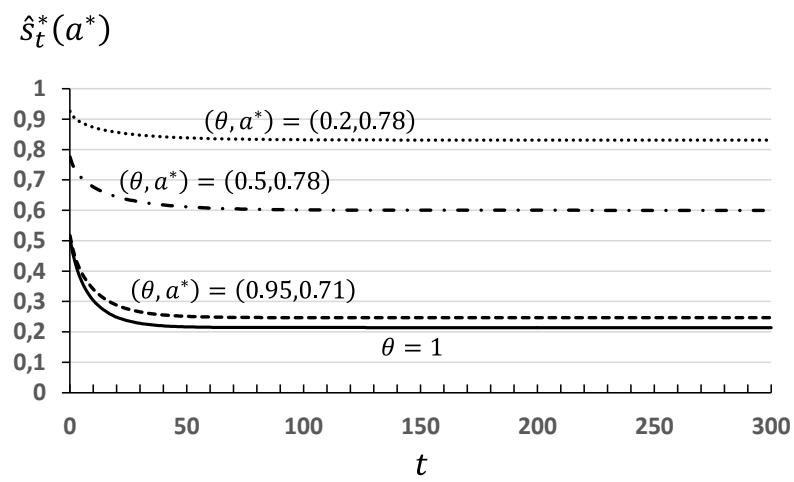

Figure 6. Equilibrium path of the saving rate

\subsection{Dynamics in Constrained Optimization Cases}

Suppose for example that individuals are endowed of preferences for consuming and bequeathing such that future generations benefit from the golden rule steady-state at time $T$, where consumption is maximized.

It is well known that for this steady-state to be possible under the standard altruistic case, the flow of Utility must be augmented to include a direct preference for wealth (or status) as for example in Zou (1994). A general specification widespread in this literature is:

$$
W\left\{c_{t}, k_{t}\right\}=\int_{t_{0}}^{\infty}\left[u_{t}\left(c_{t}\right)+v_{t}\left(k_{t}\right)\right] e^{(n-\beta) t} d t
$$


where $v_{t}\left(k_{t}\right)$ represents the part of utility derived from the capital stock, with $v_{k}>0$ and $v_{k k}<0$. Preserving same notations as in the presented paper, the resolution of the program under this assumption leads to the following rule:

$$
f_{k_{T}}=\delta+\beta-\frac{v_{k}}{u_{c}}
$$

Hence, given the ratio $v_{k} / u_{c}$ is always positive, the steady-state capital per capita in such models will always be greater than in the standard one. The alternative Utility function proposed in this paper generates a more general version of the neoclassical growth model by contrasting this result.

For a convenient comparative analysis, suppose the flow of Utility of the capitalist spirit model of Zou (1994) is given by:

$$
u_{t}\left(c_{t}\right)+v_{t}\left(k_{t}\right)=\ln \left(c_{t}\right)+\gamma \ln \left(k_{t}\right), \text { where } \gamma \in[0, \infty)
$$

For $n=0$, it can be shown that the steady-state capital per capita is given by:

$$
k_{T}=\left(\frac{\alpha+\gamma}{(\gamma+1) \delta+\beta}\right)^{\frac{1}{1-\alpha}}
$$

and is increasing in $\gamma$. The value of this parameter can be easily deduced such as to meet the golden rule steady-state.

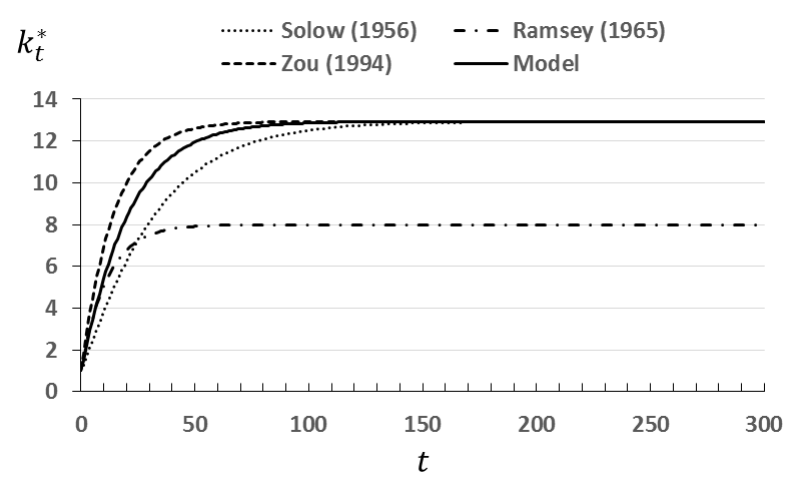

Figure 7. Equilibrium path of capital per capita

Note. Common parameters in each model are assigned the following values: $\alpha=0.3, \beta=0.02, \mathrm{n}=0, \delta=0.05, \mathrm{k}_{0}=1$. The steady-state saving rate associated to the golden rule is therefore 0.3 . In the presented model, the optimal pair $\left(\theta, \mathrm{a}^{*}\right)$ is deduced accordingly and equals (0.901, 0.85) and in the model of Zou (1994), $\gamma=0.171$. The standard Ramsey results have been included in each Figure to compare the dynamics; the steady-state level of the saving rate $\hat{\mathrm{s}}_{\mathrm{T}}^{*}$ equals 0.21 in that model.

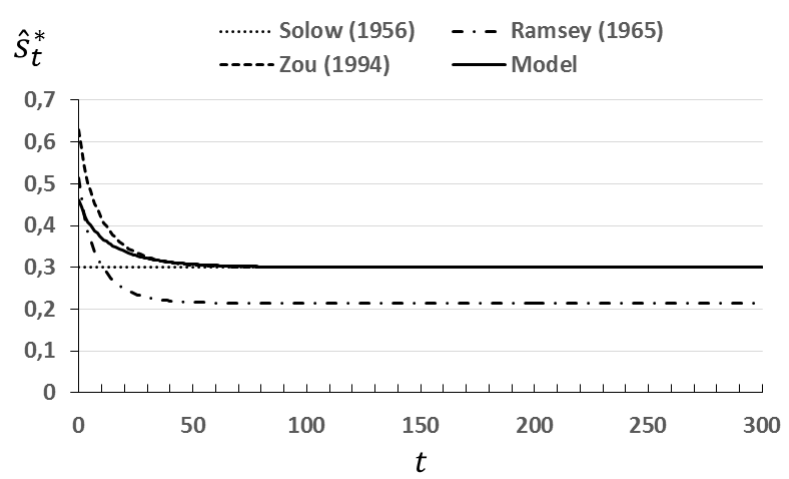

Figure 8. Equilibrium path of the saving rate

Note. Common parameters in each model are assigned the following values: $\alpha=0.3, \beta=0.02, \mathrm{n}=0, \delta=0.05, \mathrm{k}_{0}=1$. The steady-state saving rate associated to the golden rule is therefore 0.3 .

The slowest transition towards the golden rule steady-state in Figure 7 and 8, is unsurprisingly the Solow one where individuals do not take advantage of the high returns initially. In all other cases, an optimal decision implies a faster speed of growth which differs depending on the type of preferences assumed. It appears clearly that the model of Zou (1994) generates the faster transition. In other words, the stock of capital in the Utility 
function, compared to the unconsumed part of income, affects more intensively the willingness to accumulate.

An additional particularity of the proposed model shown by Figure 4, is to allow for some admissible controlled trajectories towards lower steady-states as well. Departing from the standard model, this Figure indicates that the steady-state level of capital per capita increases with the degree of preference for saving $(1-\theta)$ and with the choice parameter $a$, except for few cases where $(1-\theta)$ is relatively low. Figures 9 and 10 present an example of transition towards a lower steady-state. For a 'terminal' saving rate $\hat{s}_{T}^{*}$ of 0.15 (versus 0.21 in the standard model), two different optimal trajectories are possible. In both cases, the speed of growth remains logically greater than in the Solow model. For the case where the value of $\theta$ is higher, the corresponding value of ' $a$ ' leading to the specified steady-state with the maximum welfare is also higher and very close to one. Interestingly, the difference between the values of $a$ in each case is such that the trajectory of the individual endowed of the higher preference for consumption $\theta$, appears faster than the one who values wealth accumulation more intensively. In that case, the thriftiest individual is again less sensitive to variations of capital returns.

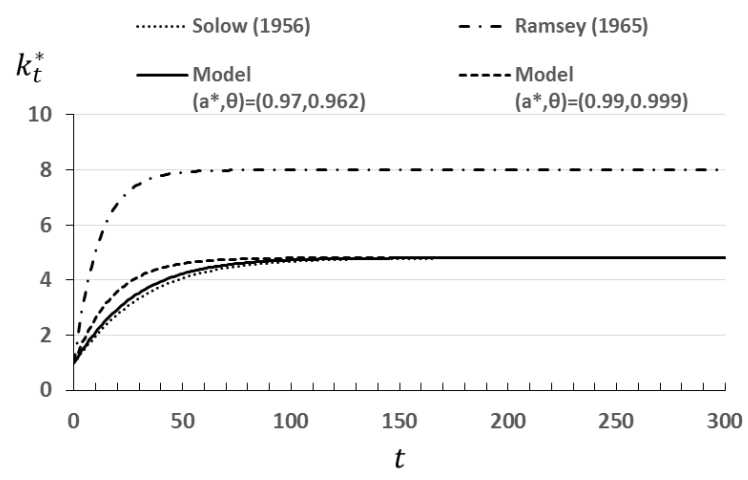

Figure 9. Equilibrium path of capital per capita

Note. Common parameters in each model are assigned the following values: $\alpha=0.3, \beta=0.02, \mathrm{n}=0, \delta=0.05, \mathrm{k}_{0}=1$. The steady-state saving rate in the Solow model and in the presented one equals 0.15 . Two solutions $\left(\theta_{\mathrm{i}}, \mathrm{a}_{\mathrm{i}}^{*}\right)$ are compatible with an optimizing behavior towards this steady-state, $(0.999,0.99)$ and $(0.962,0.97)$. The standard Ramsey results are reported for comparisons (in this model, $\hat{\mathrm{s}}_{\mathrm{T}}^{*}=$ $0.21)$.

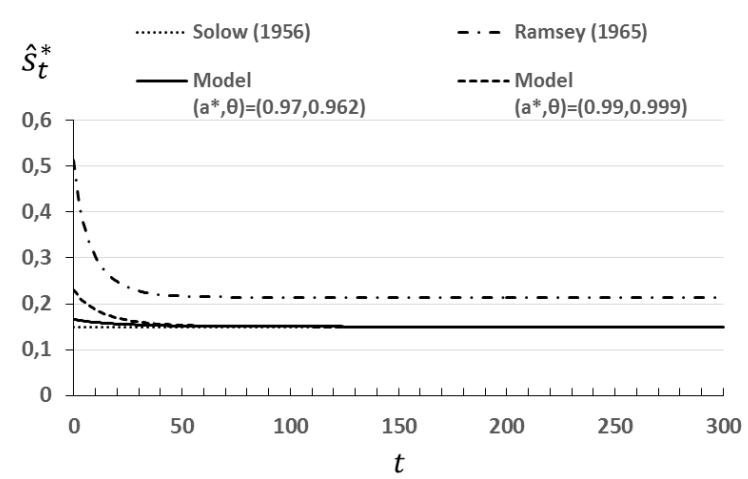

Figure 10. Equilibrium path of the saving rate

Note. Common parameters in each model are assigned the follow values: $\alpha=0.3, \beta=0.02, \mathrm{n}=0, \delta=0.05, \mathrm{k}_{0}=1$. The steady-state saving rate in the Solow model and in the presented one equals 0.15 .

\section{Conclusion}

Clearly, in the standard Ramey model, the capital accumulation occurs mechanically thanks to the interest rate. When its value is low, the incentive to save disappears. The desire to save or invest in itself is neither explicitly nor implicitly involved in the mind of the representative agent. Yet, several motives other than the interest rate are actually present in that framework. Important ones are a least the necessity to renovate the capital that depreciates, and the necessity to increase it in order to absorb a growing household. We may eventually cite also the desire to get richer as in the literature of 'capitalist spirit'. We explain in that paper that including the saving 
flow in the utility function is not only a way to make the individual humanely conscious and care about survival, but also to capture any other motives to save than the rate of return that could reasonably be involved.

The standard Ramsey model is limited in any case. It generates a steady- state level of capital per capita bounded by the golden rule level, where the resulting equilibrium saving rate reaches a maximum value of ' $\alpha$ ' (roughly estimated at 30\%), when the time preference rate tends to its lower bound level $n$. Capitalist spirit growth models as proposed for example by Zou (1994), offer a reasonable way to contrast this constraining and counterfactual property. However, the love of wealth accumulation as formalized in such models, allows to extend the set of possible dynamics to efficient and over-accumulation ones only, so that explanations of low GDP levels remain limited to the time preference rate essentially. The proposed model offers the possibility to expand the set of steady-states on both sides of the standard version, so that eventual poverty traps in low developed countries characterized by insufficient investment can also be reconciled in a same way with optimal growth theory.

In the exogenous version of Solow (1956), the individual is a saver and the desire to save is set independently of the interest rate by assumption. We show in this paper that including the saving flow in the utility function allows, not only to extend capitalist spirit models, but also to recover the properties known from the exogenous version. We even show through an additive Cobb-Douglas Utility function, that the dynamics and steady-state solution change as soon as individuals derive utility (or comfort) from saving. This should necessarily be the case if individuals are conscious about the possibility to die or hurt if they omit to save (enough) in that context. We conclude that the standard Ramsey model, used as a central structure in macroeconomic models, is actually a particular and too restrictive version of the optimal growth model where the representative individual cannot be assimilated to a real human being. Therefore, several further works are worth conducting on existing macroeconomic studies using the standard Ramsey model to check the impacts of this more appropriate dynamic structure, and hence, the robustness of the results derived from those studies in the literature.

\section{Acknowledgments}

I thank Peter Ireland for valuable comments and the distinguished mathematician Heinz Schättler for technical support in Optimal Control Theory. I also benefitted from feedback at the 3rd ISCEF held in Paris in April 2014. This paper was also part of the 82nd International Atlantic Economic Conference in Washington D.C., in October 2016.

\section{References}

Andreoni, J. (1990). Impure Altruism and Donations to Public Goods: A Theory of Warm-Glow Giving. Economic Journal, 100, 464-477. https://doi.org/10.2307/2234133

Bakshi, G., \& Chen, Z. (1996). The Spirit of Capitalism and Stock-Market Prices. American Economic Review, 86(1), 133-57.

Barro, \& Sala-i-Martin. (1995). Economic Growth. Mc Graw Hill, 1995.

Barro, R. (1974). Are Government Bonds Net Wealth? Journal of Political Economy, 82(6), 1095-1117. https://doi.org/10.1086/260266

Caroll, C. (2000). Why do the Rich Save so Much? Does Atlas Shrug? In J. B. Slemrod (Ed.), The Economic Consequences of Taxing the Rich (pp. 465-484). Harvard University Press, 2000.

Cornero, G., \& Jeanne, O. (2001). Status, the Distribution of Wealth, and Growth. The Scandinavian Journal of Economics, 103(2), 283-293. https://doi.org/10.1111/1467-9442.00245

Diamond, P. (1965). National Debt in a Neoclassical Growth Model. American Economic Review, 55, $1126-1150$.

Dynan, K., Skinner, J., \& Zeldes, S. (2002). The importance of Bequests and Life-Cycle Saving in Capital Accumulation: A New Answer. American Economic Review, 92(2), 274-278. https://doi.org/10.1257/000282802320189393

Hof, F., \& Wirl, F. (2008). Wealth Induced Multiple Equilibria in Small Open Economy Versions of the Ramsey Model. Homo Oeconomicus, 25(1), 107-128.

Kumhof, M., Rancière, R., \& Winant, P. (2015). Inequality, Leverage and Crisis. American Economic Review, 105(3). https://doi.org/10.1257/aer.20110683

Kurz, M. (1968). Optimal Economic Growth and Wealth Effects. International Economic Review, 9(3), 348-357. https://doi.org/10.2307/2556231

Kuznitz, A., Kandel, S., \& Fos, V. (2008). A Portfolio Choice Model with Utility from Anticipation of Future 
Consumption and Stock Mean Reversion. European Economic Review, 52(8), 1338-1352. https://doi.org/10.1016/j.euroecorev.2008.07.002

Laitner, J. (1992). Random Earnings Differences, Lifetime Liquidity Constraints, and Altruistic Intergenerational Transfers. Journal of Economic Theory, 58(2), 135-170. http://dx.doi.org/10.1016/0022-0531(92)90051-I

Mankiw, G., Romer, D., \& Weil, D. (1992). A Contribution to the Empirics of Economic Growth. The Quarterly Journal of Economics, 107(2), 407-437. https://doi.org/10.2307/2118477

Modigliani, F., \& Brumberg, R. (1954). Utility Analysis and the Consumption Function: An Interpretation of Cross-Section Data. In K. Kurihara (Ed.), Post-Keynesian Economics. New Brunswick, New Jersey: Rutgers University Press.

Modigliani, F. (1986). Life Cycle, Individual Thrift, and the Wealth of Nations. American Economic Review, 76(3), 297-313. https://doi.org/10.1126/science.234.4777.704

Phelps, E. (1961). The Golden Rule of Capital Accumulation. American Economic Review, 51, 638-643.

Ramsey, F. (1928). A Mathematical Theory of the Saving. Economic Newspaper, 38(152), 543-559. https://doi.org/10.2307/2224098

Samuelson, P. (1937). A Note on Measurement of Utility. Review of Economic Studies, 4(2), 155-161. https://doi.org/10.2307/2967612

Schättler, H., \& Ledzewicz, U. (2012). Geometric Optimal Control: Theory, Methods and Example. Springer Verlag, 2012. https://doi.org/10.1007/978-1-4614-3834-2

Solow, R. (1956). A Contribution to the Economic Theory of Growth. Quarterly Newspaper of Economics, 70(1), 65-94. https://doi.org/10.2307/1884513

Weber, M. (1930). The Protestant Ethic and the Spirit of Capitalism. Unwin Hyman, London \& Boston, 1930.

Yaari, M. (1965). Uncertain Lifetime, Life Insurance and the Theory of the Consumer. Review of Economic Studies, 32(2), 137-150. https://doi.org/10.2307/2296058

Zou, H. F. (1994). The Spirit of Capitalism and Long-Run Growth. European Journal of Political Economy, 10(2), 279-293. http://dx.doi.org/10.1016/0176-2680(94)90020-5

\section{Notes}

Note 1 . This idea can eventually be related to the modern literature of anticipatory feelings, like for example Kuznitz, Kandel, and Fos (2008). The individual is supposed conscious and can be affected in the present by future situations, even under a deterministic context. In our case, the thrifty individual obtains 'comfort', feels safe, or avoids anxiety when saving a part of income.

Note 2. Recall the Hurwicz-Uzawa theorem, and let the demand functions be $\xi_{\mathrm{C}}$ and $\xi_{\mathrm{S}}$, where $\mathrm{p}_{\mathrm{C}}=\mathrm{p}_{\mathrm{S}}=1$ and $\mathrm{m}$ $=\mathrm{Y}$. We have that $\xi_{\mathrm{C}}\left(\mathrm{p}_{\mathrm{C}}, \mathrm{Y}\right)$ and $\xi_{\mathrm{S}}\left(\mathrm{p}_{\mathrm{S}}, \mathrm{Y}\right)$ add up to $\mathrm{Y}$ each time and are homogenous of degree zero in prices and income. Let $\mathrm{X}$ denote the range of $\xi$. Then, the theorem states that there exists a Utility function $\mathrm{U}: \mathrm{X} \rightarrow \mathrm{R}$ on the range $\mathrm{X}$ such that $\xi_{\mathrm{C}}\left(\mathrm{p}_{\mathrm{C}}, \mathrm{Y}\right)$ and $\xi_{\mathrm{S}}\left(\mathrm{p}_{\mathrm{S}}, \mathrm{Y}\right)$ are the unique maximizers of $\mathrm{U}$ over the budget set. In other words, $\mathrm{U}$ is necessarily a function of the two desired 'activities' for which total income is shared each time.

Note 3. It can indeed be shown that the second derivative of the ' $\dot{c}_{t}=0$ locus' with respect to $\mathrm{k}_{\mathrm{t}}$ is strictly positive for $a<1$, and tends to zero when $a$ tends to 1 .

Note 4. Numerical computations of saddle paths are made according to the shooting method. A solution $a^{*}$ is considered sufficiently accurate if in its neighborhood, changes in total welfare become relatively negligible. In this part, parameters kept constant are assigned the following values: $\alpha=0.3, \beta=0.02, n=0, \delta=0.05, k_{0}=1$. For convenience, total welfare is calculated with re-scaled variables $\left(c_{t}\right.$ and $s_{t}$ are multiplied by 100).

Note 5. The golden rule of capital accumulation is indeed not necessarily what individuals prefer.

\section{Appendix A}

\section{Resolution of the dynamic program}

The social planner solves:

$$
\operatorname{Max}_{c_{t}} V\left\{c_{t}, k_{t}\right\}=\int_{t_{0}}^{\infty} U_{t}\left(c_{t}, k_{t}\right) e^{(n-\beta) t} d t
$$




$$
\text { s.t. } \quad \dot{k_{t}}=f\left(k_{t}\right)-c_{t}-(n+\delta) k_{t}
$$

Resolution for the multiplicative Cobb Douglas case:

$$
H_{t}\left(c_{t}, s_{t}\right)=c_{t}^{\theta}\left[f\left(k_{t}-c_{t}\right)\right]^{1-\theta} e^{(n-\beta) t}+\lambda_{t} e^{(n-\beta) t}\left[f\left(k_{t}\right)-c_{t}-(n+\delta) k_{t}\right]
$$

The necessary conditions:

i) $H\left(t, \lambda_{0}, \lambda_{t}, k_{t}, c_{t}\right)=\max _{v \in\left(0 ; \theta y_{t}\right)} H\left(t, \lambda_{0}, \lambda_{t}, k_{t}, v\right)$

ii) $\frac{\partial H}{\partial k}\left(t, \lambda_{0}, \lambda_{t}, k_{t}, c_{t}\right)=\frac{\partial\left[\lambda_{t} e^{(n-\beta) t}\right]}{\partial t}$

iii) $\frac{\partial H}{\partial \lambda}\left(t, \lambda_{0}, \lambda_{t}, k_{t}, c_{t}\right)=\frac{d k_{t}}{d t}=\dot{k_{t}}$

iv) $\lim _{t \rightarrow \infty} \lambda_{t} e^{(n-\beta) t} k_{t}=0$

The first order condition implies: $\partial U_{t} / \partial c_{t}=U_{c_{t}}=\lambda_{t}$. This static maximizing condition is to be substituted in the dynamical expressions that serve to derive the differential equation of the control. With $\lambda_{t} \geq 0$ and $U_{t}$ homothetic, we can express an explicit condition in a convenient way by letting $a \in(0,1)$ such that: $\lambda_{t}=$ $\left(\frac{c_{t}}{s_{t}}\right)^{\theta}\left(\frac{1-a}{a}\right) \geq 0$ and $\frac{c_{t}}{s_{t}}=\frac{a \theta}{1-a \theta}$

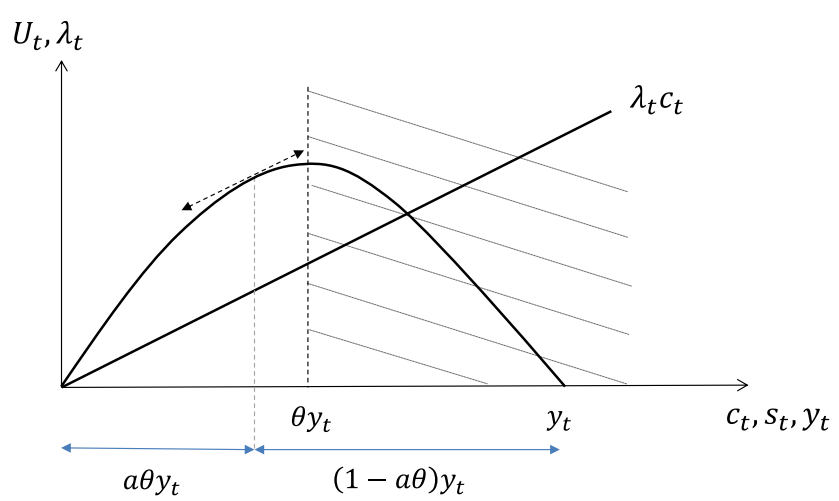

Figure A1. Static equilibrium condition in the multiplicative

As explained by Schättler and Ledzewicz (2012) p. 96, the substitution of the necessary condition for a static maximization corresponds to a weak formulation of the Pontryagin's Maximum Principle. Just like in the standard Ramsey model, it is the necessary condition that is substituted in our case (here, the equilibrium co-state and ratio).

ii) $(1-\theta)\left(\frac{c_{t}}{s_{t}}\right)^{\theta} f^{\prime}+\lambda_{t}\left[f^{\prime}-\delta-\beta\right]=-\dot{\lambda}_{t}$

$$
\begin{aligned}
& (1-\theta)\left(\frac{c_{t}}{s_{t}}\right)^{\theta} f^{\prime}+\left(\frac{1-a}{a}\right)\left(\frac{c_{t}}{s_{t}}\right)^{\theta}\left[f^{\prime}-\delta-\beta\right]=-\dot{\lambda}_{t} \\
& \left(\frac{c_{t}}{s_{t}}\right)^{\theta}\left[\left((1-\theta)+\frac{1-a}{a}\right) f^{\prime}-\frac{1-a}{a}(\delta+\beta)\right]=-\dot{\lambda_{t}}
\end{aligned}
$$

Differentiating totally condition $(i)$ :

$$
\begin{aligned}
& \frac{\partial U_{c}}{\partial c_{t}} \dot{c_{t}}+\frac{\partial U_{c}}{\partial k_{t}} \dot{k}_{t}=\dot{\lambda}_{t} \\
& \theta(1-\theta)\left(\frac{c_{t}}{s_{t}}\right)^{\theta} \frac{1}{c_{t}}\left[\left(-\frac{s_{t}}{c_{t}}-\frac{c_{t}}{s_{t}}-2\right) \dot{c_{t}}+f^{\prime}\left(1+\frac{c_{t}}{s_{t}}\right) \dot{k_{t}}\right]=\dot{\lambda_{t}}
\end{aligned}
$$

Constraining the static maximizing condition by substituting $\lambda_{t}$ means that the associated equilibrium ratio $\frac{c_{t}}{s_{t}}$ 
defined above can be constrained as well. The expression simplifies to:

$$
\theta(1-\theta)\left(\frac{c_{t}}{s_{t}}\right)^{\theta} \frac{1}{c_{t}}\left[\left(-\frac{1}{a \theta(1-a \theta)}\right) \dot{c}_{t}+f^{\prime} \frac{1}{1-a \theta} \dot{k_{t}}\right]=\dot{\lambda}_{t}
$$

Combining this expression with condition (ii) leads to:

$$
\begin{aligned}
& \frac{\theta(1-\theta)}{a \theta(1-a \theta)} \frac{\dot{c_{t}}}{c_{t}}=f^{\prime} \frac{\theta(1-\theta)}{1-a \theta} \dot{k_{t}} \frac{1}{c_{t}}+\left[\frac{1-a \theta}{a} f^{\prime}-\frac{1-a}{a}(\beta+\delta)\right] \\
& -\frac{\theta(1-\theta)}{a \theta(1-a \theta)} \dot{c_{t}}+\frac{\theta(1-\theta)}{(1-a \theta)} f^{\prime} \dot{k_{t}}=-\left[\frac{1-a \theta}{a} f^{\prime}-\frac{1-a}{a}(\beta+\delta)\right] c_{t} \\
& f^{\prime}\left[\frac{1-a \theta}{a} c_{t}+\frac{\theta(1-\theta)}{1-a \theta} \dot{k_{t}}\right]-\frac{1-a}{a}(\delta+\beta) c_{t}=\frac{(1-\theta)}{a(1-a \theta)} \dot{c}_{t}
\end{aligned}
$$

Introducing condition (iii):

$$
\begin{aligned}
& f^{\prime}\left[(1-a \theta) c_{t}+\frac{(1-\theta) a \theta}{1-a \theta} y_{t}-\frac{(1-\theta) a \theta}{1-a \theta} c_{t}-\frac{(1-\theta) a \theta}{1-a \theta}(n+\delta) k_{t}\right]-(1-a)(\delta+\beta) c_{t}=\frac{(1-\theta)}{(1-a \theta)} \dot{c_{t}} \\
& f^{\prime}\left[c_{t}\left[(1-a \theta)+\frac{(1-\theta)(1-a \theta)}{1-a \theta}\right]-\frac{(1-\theta) a \theta}{1-a \theta}(n+\delta) k_{t}\right]-(1-a)(\delta+\beta) c_{t}=\frac{(1-\theta)}{(1-a \theta)} \dot{c_{t}}
\end{aligned}
$$

The resulting dynamical system is:

$$
\left\{\begin{array}{l}
\dot{c_{t}}\left(c_{t}, k_{t}\right)=\frac{1-a \theta}{1-\theta}\left[(2-a \theta-\theta) f^{\prime}-(1-a)(\delta+\beta)\right] c_{t}-a \theta(n+\delta) f^{\prime} k_{t} \\
\dot{k_{t}}\left(c_{t}, k_{t}\right)=f\left(k_{t}\right)-c_{t}-(n+\delta) k_{t}
\end{array}\right.
$$

In other words, we know an optimal trajectory should necessarily be generated by this differential system (derived from necessary conditions). There still remain to find which static equilibrium co-state maximizes total welfare (i.e., the value of $a \in(0,1)$ ). One should understand that this value is endogenously set by the individual when choosing the preferred path.

Resolution for the additive Cobb Douglas case: $U_{t}\left(c_{t}, s_{t}\right)=\theta \ln \left(c_{t}\right)+(1-\theta) \ln \left(s_{t}\right)$

$\partial U_{t} / \partial c_{t}=0$ implies:

$\frac{\theta}{c_{t}}-\frac{1-\theta}{y_{t}-c_{t}}=\lambda_{t}$

$\frac{\theta y_{t}}{c_{t}\left(y_{t}-c_{t}\right)}-\frac{c_{t}}{c_{t}\left(y_{t}-c_{t}\right)}=\lambda_{t}$

Defining $\frac{c_{t}}{s_{t}}=\frac{a \theta}{1-a \theta}$ where $c_{t}=a \theta y_{t} \in\left[0, \theta y_{t}\right]$,

$\frac{a \theta y_{t}}{a c_{t}\left(y_{t}-c_{t}\right)}-\frac{a \theta}{c_{t}(1-a \theta)}=\lambda_{t}$

$\frac{1}{a(1-a \theta) y_{t}}-\frac{1}{(1-a \theta) y_{t}}=\lambda_{t}$

$\lambda_{t}=\frac{1-a}{a(1-a \theta) y_{t}}$

Differentiating totally the first order condition gives:

$$
\left[-\frac{\theta}{c_{t}^{2}}-\frac{1-\theta}{\left(y_{t}-c_{t}\right)^{2}}\right] \dot{c}_{t}+\frac{(1-\theta) f^{\prime}\left(k_{t}\right)}{\left(y_{t}-c_{t}\right)^{2}} \dot{k}_{t}=\dot{\lambda}_{t},
$$

The first term in brackets can for example be expressed more conveniently:

$\left[-\frac{\theta}{c_{t}^{2}}-\frac{1-\theta}{\left(y_{t}-c_{t}\right)^{2}}\right]=-\frac{\theta\left(a \theta y_{t}\right)^{2}}{c_{t}^{2}\left(a \theta y_{t}\right)^{2}}-\frac{(1-\theta)\left[(1-a \theta) y_{t}\right]^{2}}{\left(y_{t}-c_{t}\right)^{2}\left[(1-a \theta) y_{t}\right]^{2}}=-\frac{\theta}{\left(a \theta y_{t}\right)^{2}}-\frac{(1-\theta)}{\left[(1-a \theta) y_{t}\right]^{2}}$

Following same simplifying tricks, the differentiated condition can be expressed as: 


$$
\begin{aligned}
& \frac{-\theta\left(a^{2} \theta-2 a \theta+1\right)}{(a \theta)^{2}(1-a \theta)^{2} y_{t}^{2}} \dot{c}_{t}+\frac{(1-\theta) f^{\prime}}{(1-a \theta)^{2} y_{t}^{2}} \dot{k}_{t}=\frac{-(1-\theta) f^{\prime}}{(1-a \theta) y_{t}}-\frac{(1-a)}{a(1-a \theta) y_{t}}\left(f^{\prime}-\delta-\beta\right) \\
& \frac{-\left(a^{2} \theta-2 a \theta+1\right)}{a(1-a \theta)} \frac{\dot{c}_{t}}{c_{t}}+(1-\theta) f^{\prime}\left[1-\frac{(n+\delta) k_{t}}{(1-a \theta) y_{t}}\right]=-(1-\theta) f^{\prime}-\frac{1-a}{a}\left(f^{\prime}-\delta-\beta\right) \\
& \frac{-\left(a^{2} \theta-2 a \theta+1\right)}{a(1-a \theta)} \frac{\dot{c}_{t}}{c_{t}}=f^{\prime}\left[-2(1-\theta)-\frac{1-a}{a}+\frac{(1-\theta)(n+\delta) k_{t}}{(1-a \theta) y_{t}}\right]+\frac{1-a}{a}(\beta+\delta)
\end{aligned}
$$

The resulting system is therefore:

$\left\{\begin{array}{l}\frac{\dot{c_{t}}}{c_{t}}=\frac{1-a \theta}{1+a \theta(a-2)}\left[2 a(1-\theta) f_{k}+(1-a)\left(f_{k}-\beta-\delta\right)\right]-\frac{a(1-\theta)}{1+a \theta(a-2)}(n+\delta) \alpha \\ \dot{k_{t}}=f\left(k_{t}\right)-c_{t}-(n+\delta) k_{t}\end{array}\right.$

\section{Appendix B}

\section{The case of the Ak function}

Suppose $f\left(k_{t}\right)=A k$. In the Solow model, a steady-state requires necessarily $s A=n+\delta$. There is no transition dynamics (to a given intial state $k_{0}$, corresponds a steady-state level of consumption $c_{0}$ ). For concreteness, suppose $\left(A, \delta, n, k_{0}\right)=(0.07,0.05,0,10)$. We deduce the saving proportion $\mathrm{s}=0.714$ and the steady-state $\left(c^{*}, k^{*}\right)=(0.2,10)$.

Assuming now a permanent productivity shock such that A decreases to $A^{\prime}=0.065$, the new steady-state is $\left(c^{*}, k^{*}\right)=(0.15,10)$ and requires a direct increase of the saving proportion to $\mathrm{s}^{\prime}=0.769$.

In the standard Ramsey model, assuming $U=\ln \left(c_{t}\right)$, the differential system resulting from the optimization program is:

$$
\left\{\begin{array}{l}
\frac{\dot{c_{t}}}{c_{t}}=A-\beta-\delta \\
\dot{k_{t}}=f\left(k_{t}\right)-c_{t}-(n+\delta) k_{t}
\end{array}\right.
$$

The steady-state requires $A=\beta+\delta$ and $c_{t}=f\left(k_{t}\right)-(n+\delta) \cdot k_{t}$. Recalling the previous numerical values and assuming $\beta=0.02$, the initial steady-state is again $\left(c^{*}, k^{*}\right)=(0.2,10)$. Deviating slightly from this steady state with a lower capital return $A^{\prime}=0.065$ implies a suicidal behavior of the individual with a dynamics diverging towards $k_{T}=0$. Precisely, following the change in $A$, the individual choses to remain on a high level of consumption relatively to the new steady-state one, and to neglect the capital depreciation until a final state where both capital and consumption equal zero.

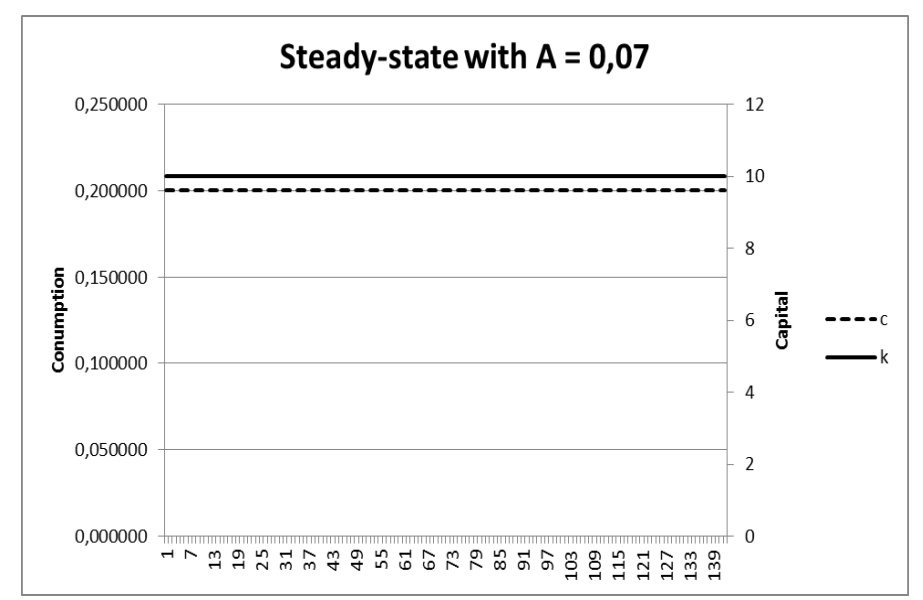

Figure B1. Steady-state levels of capital and consumption 


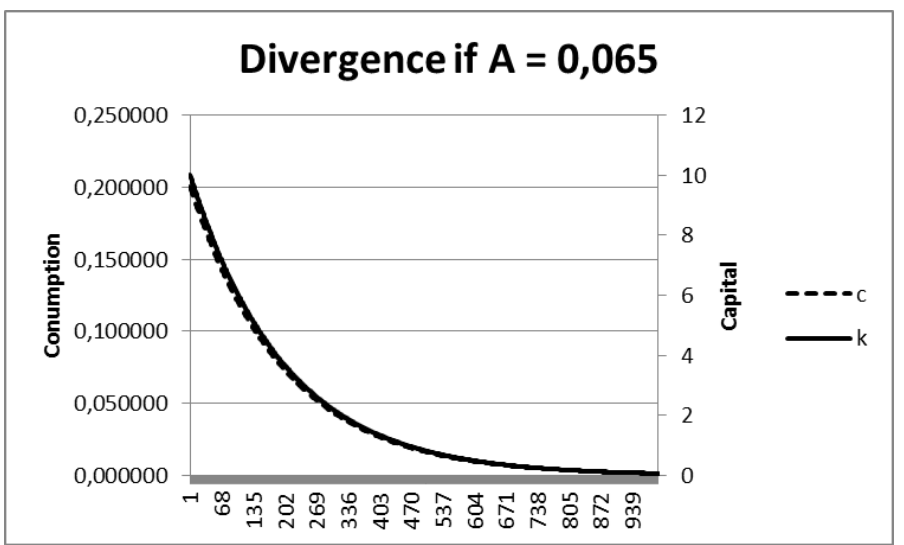

Figure B2. Divergence towards zero after a productivity shock

\begin{abstract}
A way to recover a reasonable behavior is to introduce the saving flow in the utility function. Recalling equation (9), suppose that $A=\beta+\delta$ as previously (with same numerical values). In that case, the initial steady-state chosen by the individual who maximizes total welfare is reached for $a^{*}=0.9541$. If $A$ decreases to $A^{\prime}=0.065$, the new steady-state is reached for $a^{*}=0.9484$ and corresponds to $\left(c^{*}, k^{*}\right)=(0.15,10)$.
\end{abstract}

\title{
Copyrights
}

Copyright for this article is retained by the author(s), with first publication rights granted to the journal.

This is an open-access article distributed under the terms and conditions of the Creative Commons Attribution license (http://creativecommons.org/licenses/by/4.0/). 